\title{
Stretch-shortening cycle: a powerful model to study normal and fatigued muscle
}

\author{
Paavo V. Komi* \\ Neuromuscular Research Center, Department of Biology of Physical Activity, University of Jyväskylä, P O Box 35 (LL), 40351 Jyväskylä, Finland
}

Accepted 1 March 2000

\begin{abstract}
Stretch-shortening cycle (SSC) in human skeletal muscle gives unique possibilities to study normal and fatigued muscle function. The in vivo force measurement systems, buckle transducer technique and optic fiber technique, have revealed that, as compared to a pure concentric action, a non-fatiguing SSC exercise demonstrates considerable performance enhancement with increased force at a given shortening velocity. Characteristic to this phenomenon is very low EMG-activity in the concentric phase of the cycle, but a very pronounced contribution of the short-latency stretch-reflex component. This reflex contributes significantly to force generation during the transition (stretch-shortening) phase in SSC action such as hopping and running. The amplitude of the stretch reflex component - and the subsequent force enhancement - may vary according to the increased stretch-load but also to the level of fatigue. While moderate SSC fatigue may result in slight potentiation, the exhaustive SSC fatigue can dramatically reduce the same reflex contribution. SSC fatigue is a useful model to study the processes of reversible muscle damage and how they interact with muscle mechanics, joint and muscle stiffness. All these parameters and their reduction during SSC fatigue changes stiffness regulation through direct influences on muscle spindle (disfacilitation), and by activating III and IV afferent nerve endings (proprioseptic inhibition). The resulting reduced stretch reflex sensitivity and muscle stiffness deteriorate the force potentiation mechanisms. Recovery of these processes is long lasting and follows the bimodal trend of recovery. Direct mechanical disturbances in the sarcomere structural proteins, such as titin, may also occur as a result of an exhaustive SSC exercise bout. (C) 2000 Elsevier Science Ltd. All rights reserved.
\end{abstract}

Keywords: Stretch-shortening cycle; In-vivo force measurement; Stretch reflex; Muscle damage; Neuromuscular fatigue

\section{Introduction}

The true nature of muscle function is difficult to assess from isolated forms of isometric, concentric or eccentric contractions. In real life, exercise seldom involves a pure form of these types of isolated muscle actions. The natural variation of muscle function is more often a stretch and shortening cycle and thus this model provides a good basis from which to study both normal and fatigued muscle. Two important aspects of this phenomenon are: (1) pre-activation and (2) variable activation of the muscles preceding the functional phase of a given movement (e.g. ground contact for the leg extensor muscles during running). Other important concepts that need to be addressed are length changes in muscle versus tendon

* Tel.: + 358-14-2602-073; fax: + 358-14-2602-071.

E-mail address: komi@maila.jyu.fi (P.V. Komi). during the contact phase and the role of the stretch reflex in the stretch-shortening cycle.

The stretch-shortening cycle (SSC) of muscle function comes from the observation that body segments are periodically subjected to impact or stretch forces. Running, walking and hopping are typical examples in human locomotion of how external forces (e.g. gravity) lengthen the muscle. In this lengthening phase the muscle is acting eccentrically, then a concentric (shortening) action follows. The true definition of eccentric action indicates that the muscles must be active during stretch. This combination of eccentric and concentric actions forms a natural type of muscle function called the stretch-shortening cycle or SSC (Norman and Komi, 1979; Komi, 1984; Komi and Nicol, 2000). (Fig. 1). This type of sequence in muscle function also involves the important features of preactivation and variable activation. SSC muscle function has a well-recognized purpose: enhancement of performance during the final phase (concentric action) when 


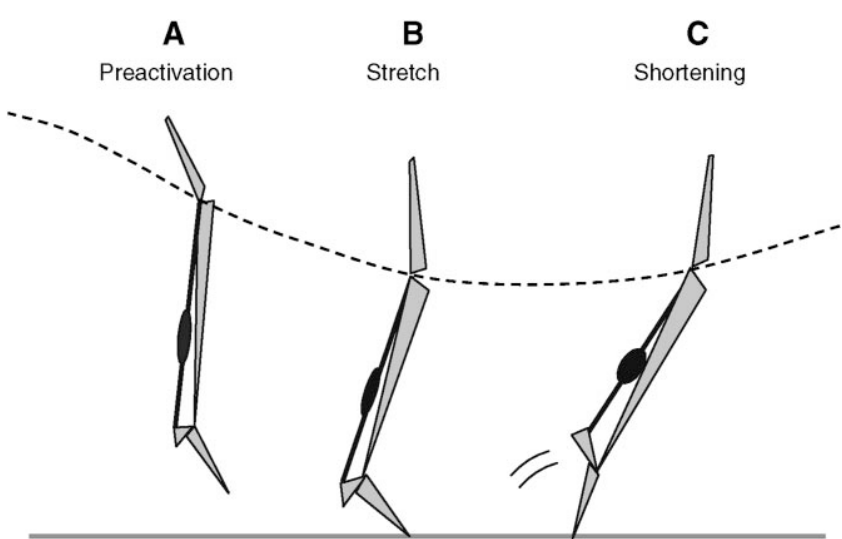

Fig. 1. In human walking, hopping and running considerable impact loads occur when contact takes place with the ground. This requires preactivation from the lower limb extensor muscles before the ground contact to make them ready to resist the impact (A) and the active braking phase (B). The stretch phase is followed by a shortening (concentric) action (C) (adapted from Komi, 1984).

compared to the isolated concentric action. This can be demonstrated in isolated preparations with constant electrical stimulation (e.g. Cavagna et al., 1965, 1968), in animal experiments with natural and variable muscle activation (e.g. Gregor et al., 1988) and in maximal effort conditions of human SSC actions (Cavagna et al., 1968; Komi, 1983). Considerable effort has been devoted to explain the mechanisms for force and power potentiation during a SSC. Cavagna et al. (1965) was one of the first to argue that this enhancement is primarily from stored elastic energy. Since that time many additional alternative explanations (e.g. Huijing, 1992; Van Ingen-Schenau et al., 1997; Komi and Gollhofer, 1997) have been presented. However, no convincing evidence has been presented that negates elasticity as an important element in force potentiation during a SSC.

The schematic presentation of Fig. 1 takes into consideration the common assumption that in a SSC the contractile and tensile elements are stretched during the eccentric phase. There are, however, arguments in the literature suggesting that the contractile component may maintain a constant length (Hoff et al., 1983; Belli and Bosco, 1992) or even shorten (Griffiths, 1991) during the early phase of ground contact.

The present report reviews the work of SSC muscle actions performed during human experiments primarily in our laboratory. The main focus will be to demonstrate with in vivo measurements the recoil nature of a SSC and how the stretch-reflex can play an important role in force potentiation. The SSC model will then be introduced for fatigue experiments where it's unique loading characteristics can be used to examine neuromuscular fatigue in a very comprehensive way.

\section{Use of in vivo force measurements to characterize the SSC in human locomotion}

Two techniques can be applied to record directly, and in vivo, tendon forces in humans: a buckle transducer method and an optic fiber technique. From these methods, the buckle technique is a more invasive one and it was used solely for Achilles tendon (AT) force recordings (e.g. Komi et al., 1987; Komi, 1990; Fukashiro et al., 1993, 1995). The buckle is surgically implanted around the AT under local anesthesia, but the subject is able to perform $2-3 \mathrm{~h}$ of unrestricted locomotion including walking, running (at different speeds), hopping and jumping. In some cases even maximal long jumps were performed without any discomfort (Kyröläinen et al., 1989). Fig. 2 presents a typical recording obtained during running at a moderate speed. There are several important features to be noted in this figure. First, the changes in muscle-tendon length are very small (6-7\%) during the stretching phase. This suggests that the conditions favor the potential utilization of short-range elastic stiffness (SRES) (Rack and Westbury, 1974) in the muscle. Various length changes are reported in the literature demonstrating that effective range of SRES in in vitro preparations is $1-4 \%$ (e.g. Ford et al., 1978; Huxley and Simmons, 1971). In the intact muscle tendon, in vivo, this value is increased because series elasticity and fiber geometry must be taken into account. This could then bring the muscle-tendon lengthening to $6-8 \%$. When measurements are made at the muscle fiber level the values could be smaller naturally, as shown by Roberts et al. (1997) in turkeys running on level ground.

The buckle technique introduced the basic behavior of the human AT-triceps surae complex during SSC activities (Komi, 1990; Fukashiro et al., 1993). Surprisingly, the technique revealed that also in bicycling a small, but meaningful, SSC action could be identified for both gastrocnemius and soleus muscles (Gregor et al., 1991). The method also provided the basis of analyzing the instantaneous force-velocity curves during various SSC activities (see Fig. 4).

The buckle method requires a considerable surgical procedure and a long healing time before normal locomotion can be resumed after the measurements. These limitations can be overcome by a novel optic fiber technique introduced recently (Komi et al., 1996). This technique is based on light intensity modulation when a thin optic fiber is compressed inside the tendon. The fiber is usually $0.5 \mathrm{~mm}$ in diameter and has a polymethol metachrylate core and a fluorinated polymer cladding. The fiber can be inserted into a variety of tendons and ligaments. Fig. 3 presents how the optic fiber is put in place. First, a sterile 19 gauge needle is passed through the tendon. The optic fiber, sterilized with ethyl oxide at $37^{\circ} \mathrm{C}$, is then passed through the needle. By removing the needle the fiber remains in situ. After the insertion, both 


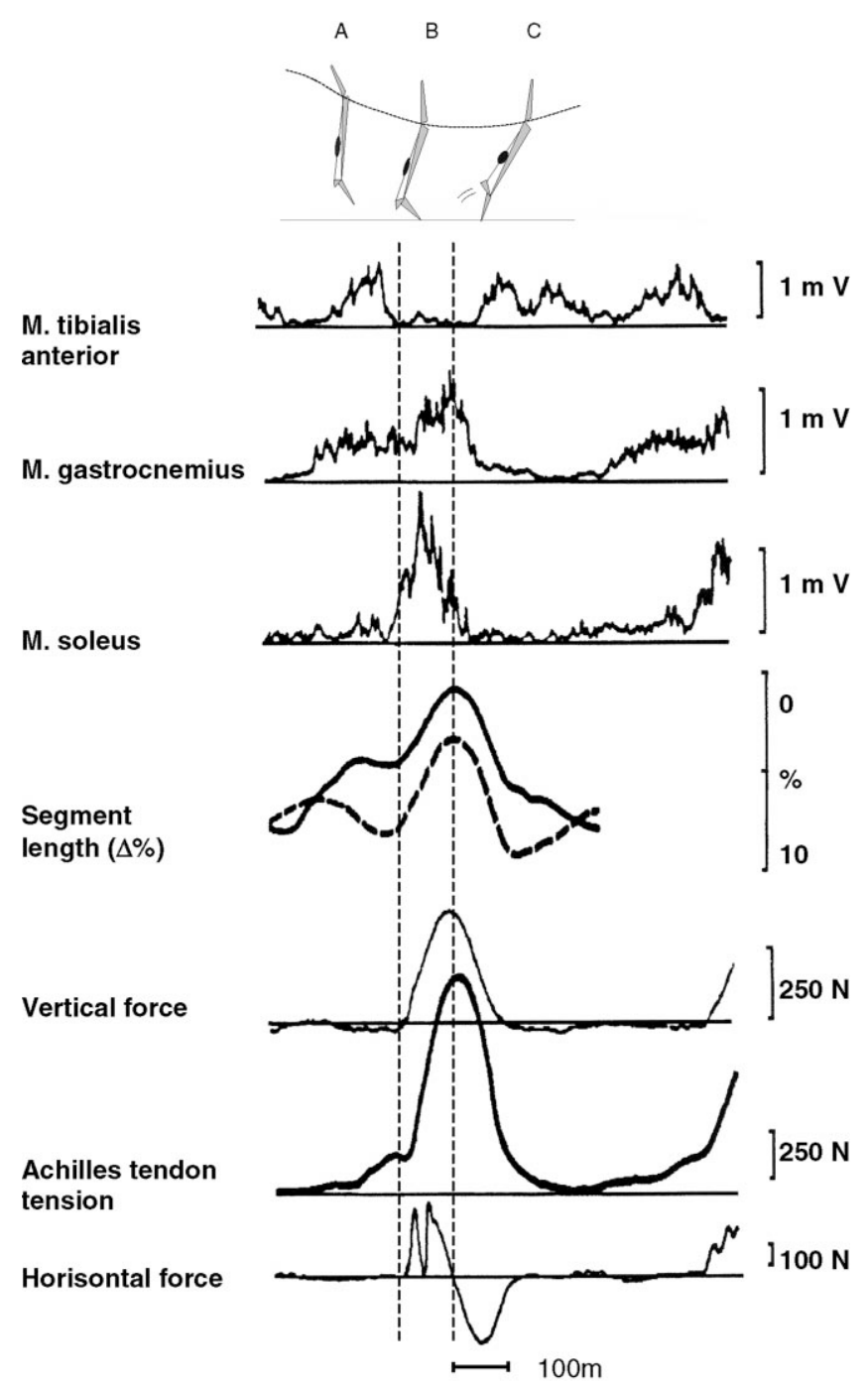

Fig. 2. Demonstration of SSC for the triceps surae muscle during the (functional) ground contact phase of human running. Top: Schematic position representing the three phases of SSC presented in Fig. 2. The rest of the curves represent parameters in the following order (from top to bottom): Rectified surface EMG records of the tibialis anterior gastrocnemius and soleus muscles, segmental length changes of the two plantar flexor muscles (dashed = soleus, solid = gastrocnemius), vertical ground reaction force, directly recorded Achilles tendon force, and the horizontal ground reaction force. The vertical lines signify, respectively, the beginning of the foot (ball) contact on the force plate and the end of the eccentric phase. The subject was running at moderate speed, (Komi, 1992).

ends of the fiber are carefully smoothed and cleaned before connecting them to the small unit containing the transmitter-receiver parts as well as relevant amplifiers. Depending on the tendon or ligament under study different calibration procedures can be applied. In all cases the response of the optic fiber precisely follows the external loads used (Komi et al., 1995; Arndt et al., 1998; Finni et al., 1998).

The force-velocity (FV) relationship (Hill, 1938) describes the fundamental mechanical properties of human muscle. Its direct application to natural locomotion, such

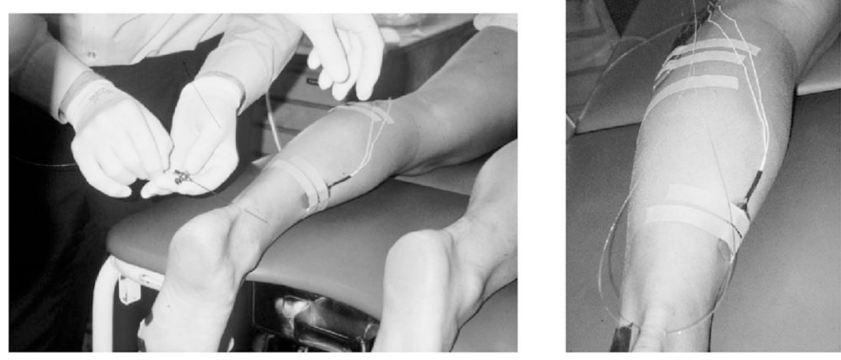

Fig. 3. Demonstration of the insertion of the optic fiber into the tendon. Left: After the 19 gauge needle has been inserted through the tendon, the $0.5 \mathrm{~mm}$ thick optic fiber is thread through the needle. The needle is then removed and the optic fiber remains in situ inside the tendon (right).
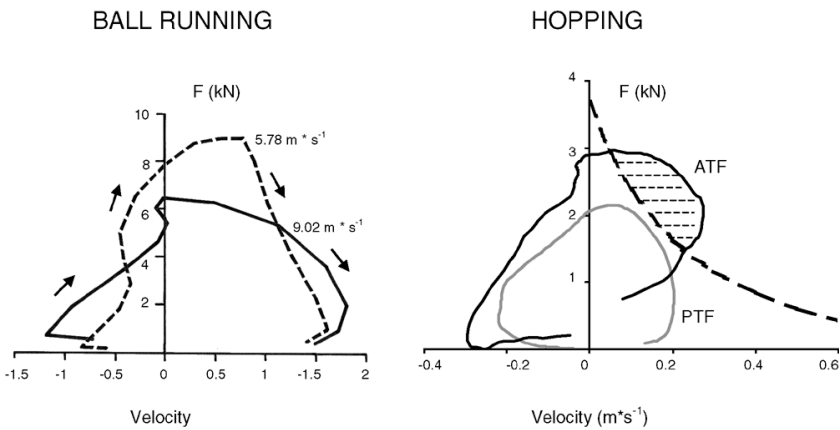

Fig. 4. Examples of instantaneous force-velocity curves measured in human running (left) and hopping (right). The records on the left were obtained with the buckle transducer (Komi, 1992) and those on the right with the optic fiber (Finni et al., 1998). Each record is for a functional (contact) phase on the ground. In each curve the upward deflection signifies stretching (eccentric actions) and the downward direction shortening (concentric action) of the muscle tendon complex during ground contact. The velocity axis has been derived from segmental length changes according to Grieve et al. (1978).

as SSC, may however, be difficult due to the necessity of in situ preparations to utilize constant maximal activations. When measured in vivo during a SSC, the instantaneous force-velocity curve (Fig. 4) is very different from the classical curve obtained for pure concentric actions with isolated preparation (Hill, 1938) or with human forearm (Wilkie, 1950; Komi, 1973). The buckle experiment (Fig. 4, left) did not include comparative records obtained in a classical way, but the form of the FV curves suggest considerable force potentiation in the concentric phase. Our recent experiments with the optic fiber technique, although not yet performed at high running speeds, suggest similar potentiation. The right side of Fig. 4 shows simultaneous plots for both patella and AT forces during hopping. The records signify that in short contact hopping the triceps surae muscle behaves in a bouncing ball-type action (see also Fukashiro et al., 1987, 1993). When the hopping intensity is increased or 
changed to counter-movement-type jumps, the patella tendon force increases and the AT force may decrease (Finni et al., 1998). The classical type of curve obtained with constant maximal activation for an isolated concentric action is also superimposed in the same graph with the AT force (Fig. 4 right). The shaded area between the two AT curves suggests a remarkable force potentiation for this submaximal effort. These findings are essentially similar to those obtained by Gregor et al. (1988), who measured mechanical outputs of the cat soleus muscle during treadmill locomotion. In their study the force generated at a given shortening velocity during the late stand phase (corresponds to the concentric part in Fig. 4) was greater, especially at high speeds of locomotion, than the output generated at the same shortening velocity in situ. Thus, both animal and human in vivo force experiments seem to give similar results with regard to the force-velocity relationships during a SSC.

The difference between the instantaneous force-velocity curve and the classical may be partly due to natural differences in muscle activation levels between the two types of recordings. In situ preparations may measure primarily the shortening properties of the contractile elements in the muscle. However, natural locomotion, primarily utilizing a SSC action, involves, controlled release of high forces caused mainly by the eccentric action. This high force favors storage of elastic strain energy in the muscle-tendon complex. A portion of this stored energy can be recovered during the subsequent shortening phase and used for performance potentiation. Thus, natural locomotion with, primarily, SSC actions may produce efficient muscle outputs which can be very different from the conditions of isolated preparations (where activation levels are held constant and storage of strain energy is limited). It needs to be emphasized that in SSC activity, such as running or hopping (Fig. 4), the muscle activation usually peaks before the eccentric phase ends.

Both of these in vivo techniques have certain limitations. While the transducers measure reliably and directly the forces in the tendon, they do not give simultaneous records of the length changes in the muscle-tendon complex. These must be estimated using high-speed video and appropriate anatomical models (e.g. Frigo and Pedotti, 1978; Grieve et al., 1978). These calculations then need to be synchronized with the tendon force data. The obtained results and relationships cannot, however, be used to generate simultaneously information about: (1) the change in length of the muscle fibers, (2) the change in the fiber orientation with the line of force application, and (3) the change in length of the tendinous compartment. Successful in vivo recordings of changes in muscle fiber length have been made in animal models, such as in cat walking (Griffiths, 1991) and turkey running on a treadmill (Roberts et al., 1997).
The common assumption has been that in SSC activities both the muscle fiber compartment and the tendon would change their lengths in phase. This assumption has recently been challenged, because the muscle fibers may stay at a constant length (Belli and Bosco, 1992) or they can even shorten (e.g. Griffiths, 1991) while the whole muscle-tendon complex is lengthening. Although this problem needs to be studies further, especially in human locomotion, the force-velocity curves presented in Fig. 4 must be interpreted more correctly to refer to the function of the muscle-tendon as one entity.

\section{Can stretch-reflexes contribute to force enhancement during SSC?}

Hopping and running, activities which are often used as models of a human SSC, seem very suitable for possible interaction from stretch reflexes. These activities seem very effective due to the following fundamental conditions (Komi and Gollhofer, 1997): (1) the muscles are preactivated before touch down (and the braking phase) (see Fig. 2); (2) the eccentric (lengthening phase) is short and fast, and (3) there is an immediate transition (Short delay) between stretch (eccentric) and shortening (concentric) phases. Because stretch reflexes play an important role in stiffness regulation (Hoffer and Andreassen, 1981), their possible interaction in SSC activities could mean a net contribution to muscle stiffness already during the eccentric part of a SSC.

It is difficult to imagine that proprioceptive reflexes would not play any significant role in human locomotion such as a SSC. Their possible role has, however, been questioned (Van Ingen-Schenau et al., 1997) by an argument that the time constraints limit their possibility to have any functional meaning during the stance phase of, for example, running. It is obvious that in normal movements with high EMG activity the magnitude and net contribution of reflex regulation of muscle force are methodologically difficult to assess. The task becomes much easier when one studies relatively slow (1.2-1.9 $\mathrm{rad} \mathrm{s}^{-1}$ ) passive dorsiflexions. In this condition the stretch-induced reflex EMG has been reported to enhance AT force by $200-500 \%$ over the pure passive stretch without a reflex EMG response (Nicol and Komi, 1998). Fig. 5 is an example of these measurements and it shows a typical delay of $10-12 \mathrm{~ms}$ between the onset of reflex EMG and onset of force potentiation.

This time delay is similar to electrical stimulation measurements performed together with optic fiber recordings of AT force (Komi et al., unpublished observation). Considering the duration of a simple stretch reflex loop of $40 \mathrm{~ms}$, the maximum delay between initial stretch and subsequent force potentiation would be around 50-55 ms. When referred to running, the first contact on the ground would indicate the point of initial stretch. In 

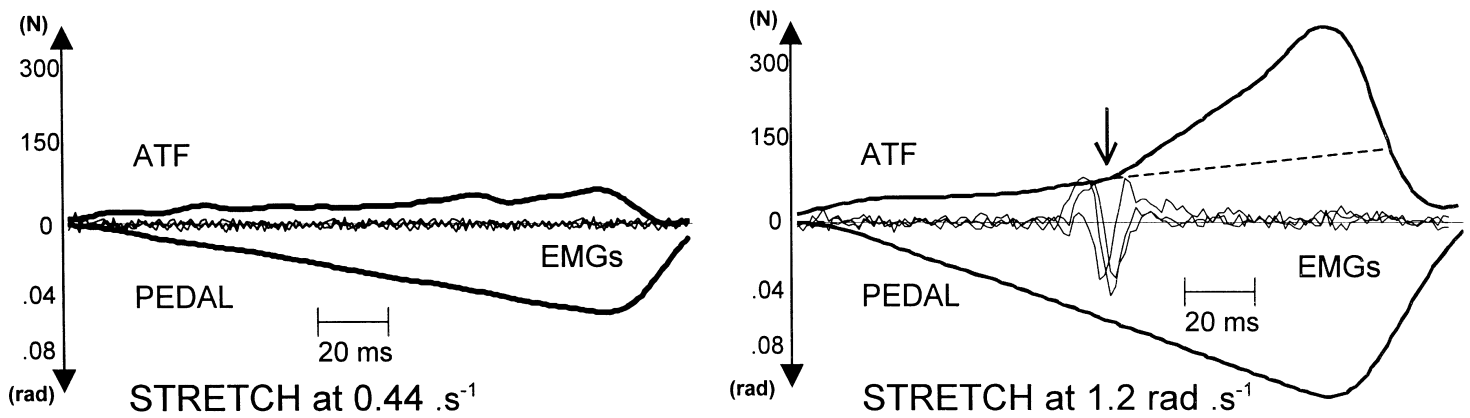

Fig. 5. Demonstration of passively induced stretch reflexes on the Achilles tendon force (ATF). Left: passive dorsiflexion at slow stretch caused no reflex EMG response and led to a small and rather linear increase of the ATF (pure passive response). Right: in case of faster and larger stretches the reflex contribution to ATF corresponds to the additional AFT response above the pure passive influence represented by the dashed line. The arrow indicates the beginning of the reflex-induced force increase. The time lag between the onset of EMG and onset of force increase is 10-12 ms (Nicol and Komi, 1998).

marathon running, the contact phase typically lasts almost $250 \mathrm{~ms}$, implying that this reflex-induced force enhancement would have functional meaning already during the eccentric phase of the cycle. As the contact phase duration (braking and push off) decreases as a function of the running speed (Luhtanen and Komi, 1978), the net reflex contribution will occur at the end of the eccentric phase at faster speeds. In addition, it may be extended partly to the push-off phase in maximal sprinting, where the total contact time is only about $90-100 \mathrm{~ms}$ (Mero and Komi, 1985). These time calculations confirm that stretch reflexes could have ample time to be instrumental in force and power enhancement during a SSC. This includes even in most cases the latter part of the eccentric phase. Thus, there are no time constraints for reflexes to be operative in stiffness regulation during a SSC. The reflex components identified in Fig. 6 represent a common observation which is characteristic to running and hopping. The short-latency component, appearing about $40 \mathrm{~ms}$ after the ground contact, is visible in all examined muscles and is especially strong in the soleus muscle (Fig. 6). These records were obtained by averaging the rectified EMGs over several trials involving two leg hops with contact times. Voigt et al. (1997) obtained similar results also with short contact hops. They also calculated the origin-to-insertion muscle length and fiber length changes. From these data, it was concluded that the stretches were high enough during the early contact phase to induce sufficient muscle spindle afferent activation. Appearance of these reflex components is a very common and repeatable observation. By combining the observation of isolated measurements from Fig. 5 and those in the SSC activity from Fig. 6 one can be confident that the reflexes are contributing to the efficiency of motor output by making the force output more powerful. In a SSC this can be accomplished proficiently by an immediate and smooth transfer from the preactivated and eccentrically stretched muscle tendon complex to the concentric push-off (in case of running or hopping).

\section{BILATERAL HOPPING}

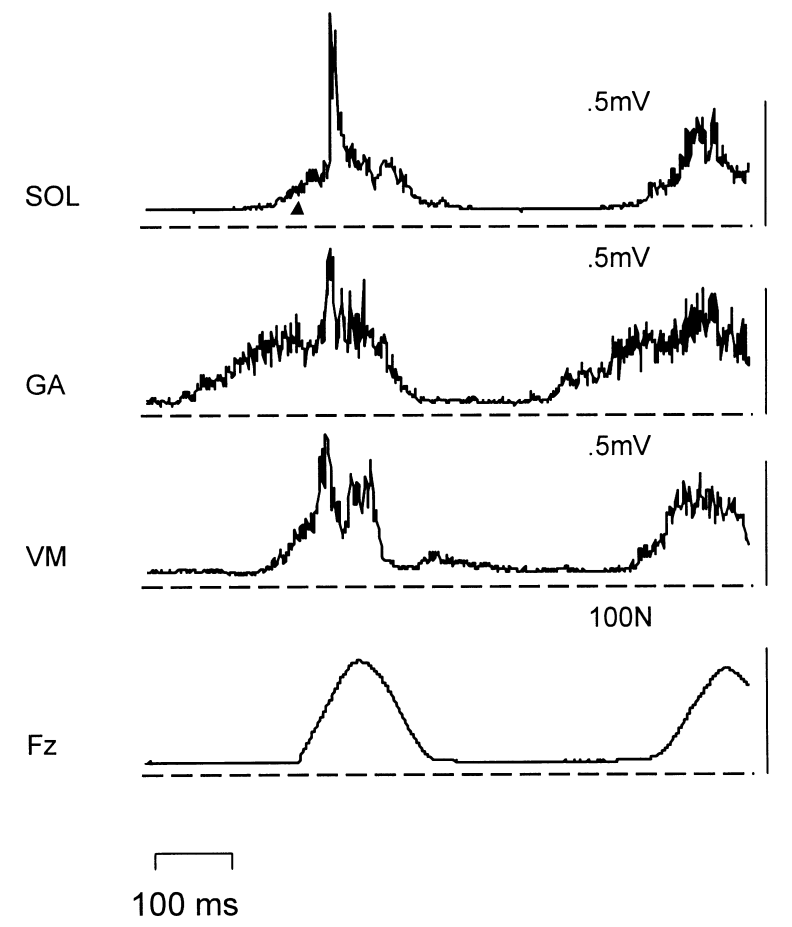

Fig. 6. Short contact SSC-type hopping introduces clear bursts in rectified EMG records. This representative example is from a drop jump performed from $60 \mathrm{~cm}$ height. Timing of the sharp EMG reflex peak occurs within $40-45 \mathrm{~ms}$ after the initial ground contact corresponding to a short latency component (SLC) in the classification of Lee and Tatton (1982). The reflex peak in these jumps is usually very clear in the soleus (SOL) muscles, but can be identified for the gastrocnemius (GAS) and vastus medialis (VM) muscles as well. FZ signifies the vertical ground reaction force (from Komi and Gollhofer, 1997).

It can be assumed that before ground contact in a SSC the initial lengthening of the muscle tendon complex of Fig. 6 occurs in the more or less compliant Achilles tendon. As soon as the "critical" tension in achieved, which is determined by the amount of activity (preactivation) sent to the muscles prior to contact, the forceful 
"yielding" of the cross-links of the acto-myosin complex may take place with concomitant loss of potential energy stored in the lengthened cross-bridges (e.g. Flitney and Hirst, 1978). From in vitro studies it is known that yielding of active cross-links can be prevented by intense muscular activation. This preventative intense muscular activation could be monitored most effectively by the stretch-reflex system that is highly sensitive to the length and tension changes in the muscle-tendon complex. As explained earlier muscle activity from a stretch-reflex response could occur as early as the latter part of the eccentric phase. The latencies of 40-45 and 10-14 ms for the reflex loop and electromechanical delay, respectively, fit well with the occurrence of short and medium latency stretch-reflex components (e.g. Lee and Tatton, 1982). Our recent data on combined stretch and reflex potentiation (Nicol and Komi, 1998) are well in agreement with the short range elastic stiffness (SRES) concept demonstrating that the cross-bridge force resistance to stretch is particularly efficient during the early part of the crossbridge attachment (Edman, 1980). Therefore, the rapid reflex-induced cross-link formation could play a substantial role in the force generation during stretch.

Thus, there seems to be enough evidence that stretch reflexes play an important role in a SSC and contribute to force generation during the touch down phase in activities such as running and hopping. Depending on the types of hopping, for example, the amplitude of the short latency stretch-reflex peak and its force increasing potential may differ considerably. However, the combination of the "pre-reflex" background activation and the following reflex activation might represent a scenario that supports yield compensation and fast rate of force development (Voigt et al., 1998).

\section{SSC is a unique model to study neuromuscular fatigue}

The mechanisms presented above are not only relevant in non-fatigued situations and can be put under severe stress during SSC fatigue. In traditional fatigue experiments, with either isometric or concentric actions, the fatigue effects can be discussed primarily from a metabolic point of view. In SSC fatigue impact loads are repeated over a certain time period with the exercise taxing all the major elements: metabolic, mechanical, and neural. It is SSC fatigue models, in particular, which cause disturbances in stretch-reflex activation and, consequently, provide an excellent basis for studying muscle function.

Evidence has been presented that both short- and long-duration fatiguing exercises lead to a deteoriation in neuromuscular performance. SSC fatigue usually results in a reversible muscle damage process and has considerable influence on muscle mechanics, joint and muscle stiffness and reflex intervention.
There are several models to study exhaustive SSC exercise, but they have all given remarkably similar results. A special sledge ergometer developed in our laboratory (Kaneko et al., 1984; Komi et al., 1987) has been used to perform short-duration SSC exercises to induce fatigue in either arm (Gollhofer et al., 1987) or leg muscles (Nicol et al., 1996; Horita et al., 1996; Avela and Komi, $1998 \mathrm{a}, \mathrm{b})$. Another possibility is to use long-duration exercise, such as marathon running, as the SSC fatigue model. In these different studies, the immediate changes in mechanical performance clearly reveals a loss of tolerance to imposed stretch loads. Fig. 7 is an example of an arm exercise (Gollhofer et al., 1987) in which 100 SSC were characterized by a progressive increase in the contact time in both the braking and push-off phases. More noticeable, however, was the progressive increase in impact spike force and a coincidental decrease in post-impact spike force.

The marathon run model has also shown similar changes in the ground contact force parameters. This includes submaximal running tests (Komi et al., 1986) and submaximal and maximal SSC tests (Nicol et al., 1991a, b). Fig. 8 is a representative example of such a result which has been confirmed in subsequent tests with a similar marathon run model (Avela et al., 2000b). Kinematic analysis has revealed that these ground reaction force changes are associated with problems to maintain constant angular displacement during contact when fatigue progresses. In a fatigue state the drop in the force after the impact is likely to be related to the observed faster and longer flexion movement (Nicol et al., 1991a, b; Horita et al., 1996). In case of the arm exercises the

\section{SUBMAXIMAL FORCE}

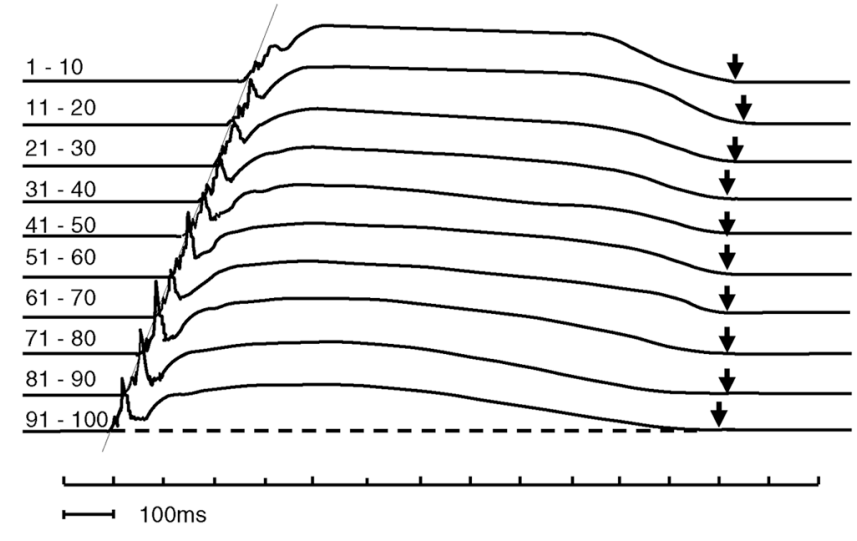

Fig. 7. Fatiguing arm SSC exercise resulted in progressive changes in the recorded reaction force during the hand contact with the sledge force plate. The records have been averaged for groups of 10 successive force-time curves. Note the increase in the impact spike with a coincidental decrease in post-impact and spike force when fatigue progressed. The dropping height was kept constant during the whole series of drops (Gollhofer et al., 1987). 


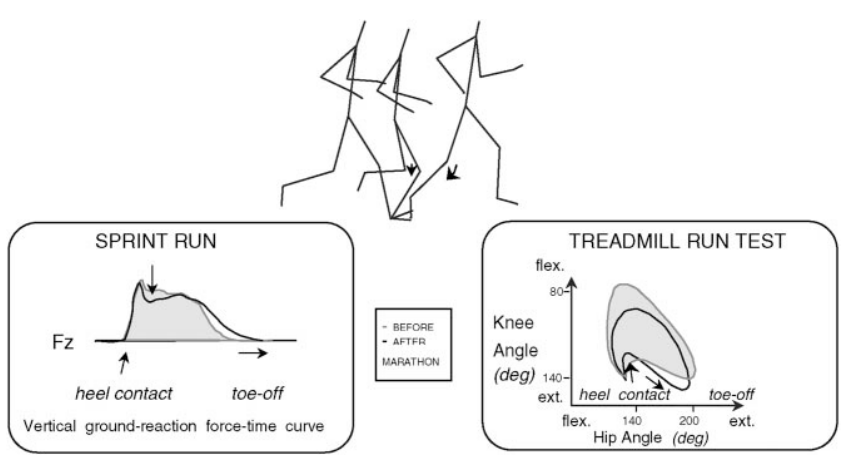

Fig. 8. Influence of a marathon run on the vertical ground reaction force (left) and on the knee/hip angle-angle diagram (right). Notice the sharp drop in the peak of the sprint force-time curve (left) after the marathon. The angle/angle diagram shows greater knee flexion immediately after heel contact in the post marathon situation (adapted from Nicol et al., 1991a, b).

dramatic increase in the impact peak results,most likely, from increased preactivity of the arm extensors (Gollhofer et al., 1987). The drop in force after impact is, however, probably the important indicator of reduction in tolerance to repeated stretch loads as fatigue progresses. A logical consequence of this is that in order to maintain the same SSC performance, for example at a constant marathon speed, the subject must perform greater work during the push-off phases leading to even a faster progression of fatigue.

The mechanical effects of fatiguing SSC exercise have also long- lasting consequences which are, in many ways, similar to pure eccentric exercise. Eccentric fatigue has, however, been referred to quite extensively previously (e.g. Clarkson et al., 1992; Komi and Nicol, 2000). Therefore, it will not be discussed in any detail at this time.

Recovery from SSC fatigue is a delayed process and takes place in parallel between maximal EMG activation and maximal force (Avela et al., 2000b). A more detailed examination of the recovery process, especially from short-duration intensive SSC exercise, indicates that it takes place in a bimodal fashion. This bimodal trend involves a dramatic decline immediately after the exercise followed by a short-lasting recovery and then a subsequent secondary drop. This second decline in performance may peak either around the 2nd or 3rd day post-exercise (Nicol et al., 1996; Avela et al., 1999a; Horita et al., 1999). The immediate reduction in performance is naturally related primarily to metabolic disturbances, whereas the secondary decline must be associated with the well-known inflammatory process related to muscle damage (Faulkner et al., 1993).
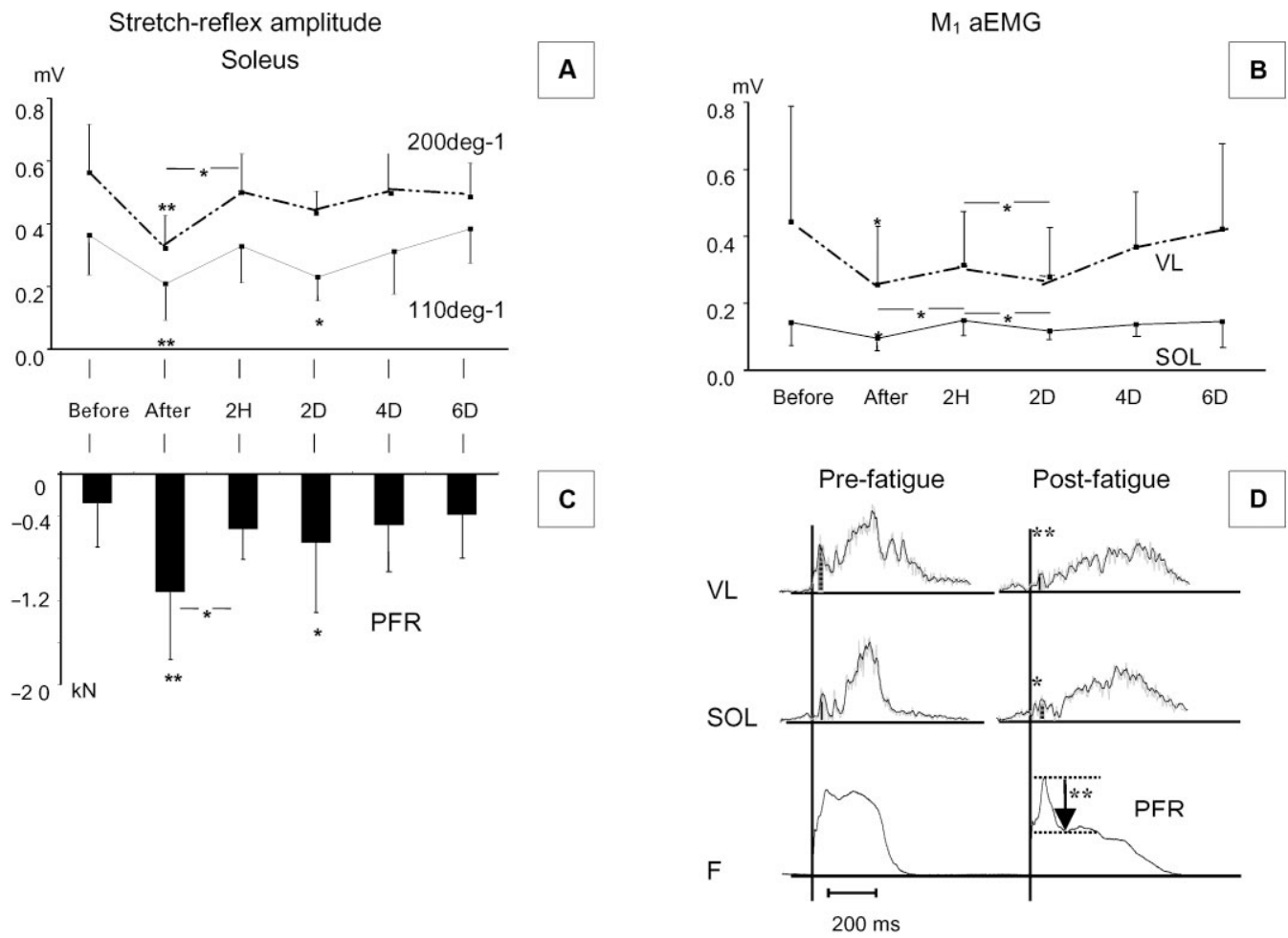

Fig. 9. Bimodal trend of recovery of stretch-reflexes and ground reaction force. The stretch-reflexes were measured in two different tests in a group of seven runners before and after a marathon run. A: mean changes in the peak-to-peak stretch-reflex amplitude of the soleus muscle recorded during 10 mechanically induced passive dorsiflexions ( $0.17 \mathrm{rad}$ induced at 1.9 and $\left.3.5 \mathrm{rad} \cdot \mathrm{s}^{-1}\right) \mathrm{B}$ : Mean values (S.D.) of average area of the SLC component (M1 ${ }_{\mathrm{a}} \mathrm{EMG}$ ) of Sol and VL muscles, and C: peak force reduction (PFR) measured during the standard sledge jump tests. The parameters in B and C are also shown as pre- and post-marathon comparisons in D. Please note the coupling in the reduction and recovery between reflex parameters and PFR. (Avela et al., 1999a) 

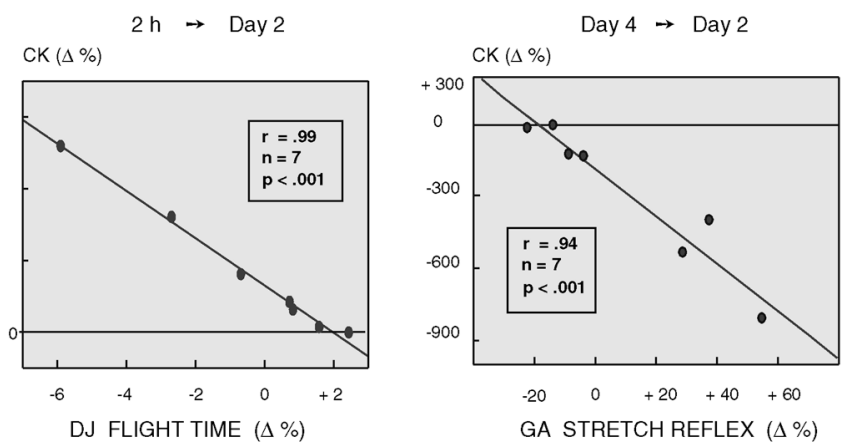

Fig. 10. Left: increase in CK activity during the first two days after exhaustive SSC exercise may be associated with a decrease in the drop jump performance. Right: a similar association is also possible between the recovery of CK activity and stretch-reflex amplitude as measured between days 2 and 4 post SSC fatigue (from Nicol et al., 1996).

Sensitivity of the stretch-reflex to passive perturbation is dramatically reduced after exhaustive SSC fatigue (Nicol et al., 1996). The same phenomenon has now been repeatedly observed during the SSC activity itself (Horita et al., 1996; Avela and Komi, 1998a, b; Avela et al., 1999a). The recovery is long lasting and follows again the bimodal trend in parallel with the mechanical parameters. Fig. 9 shows this parallelism as a representative example. The recovery process is further delayed when the same exhaustive fatigue protocol is repeated, before full recovery, on days 5 and 10 after the first exercise bout (Nicol et al., 1994). This implies that disturbed stiffness regulation needs a very long time to resume a normal status after exhaustive SSC exercise.
There seems to be enough evidence to suggest that coupling could also exist between the performance reduction in SSC and the inflammatory processes resulting from muscle damage. Firstly, decreases in SSC performance is related to increases in an indirect plasmatic marker (creatine kinase (CK) activity) of muscle damage in the phase corresponding to secondary injury (Faulkner et al., 1993) and shown in Fig. 10 (left). This coupling concept is further emphasized on the right part of Fig. 10 which shows that the subsequent reduction in CK activity between days 2 and 4 post-exercise is also related to the respective recovery of the peak-to-peak stretch-reflex EMG amplitude of the examined muscle. This clearly implies that stiffness regulation itself behaves in a similar manner. Horita et al. (1996) have demonstrated that a parallelism exists between the fatigue induced changes in the stiffness parameters and the short latency stretch-reflex component (SLC).

Fig. 11 summarizes our current view of the possible interactions which deal with the coupling between muscle damage, reduced stretch-reflex sensitivity, reduced stiffness regulation and, finally, with deteriorated SSC performance. Both presynaptic inhibition (III and IV afferent activation and possibly GTO activation) and several processes of disfacilitation of the alpha motoneuron pool may be involved in coupling. In the latter processes (disfacilitation), our current data rule out the possibility for meaningful influences of reduced fusimotor support to the muscle. Instead, they strongly suggest that the muscle spindle could be directly
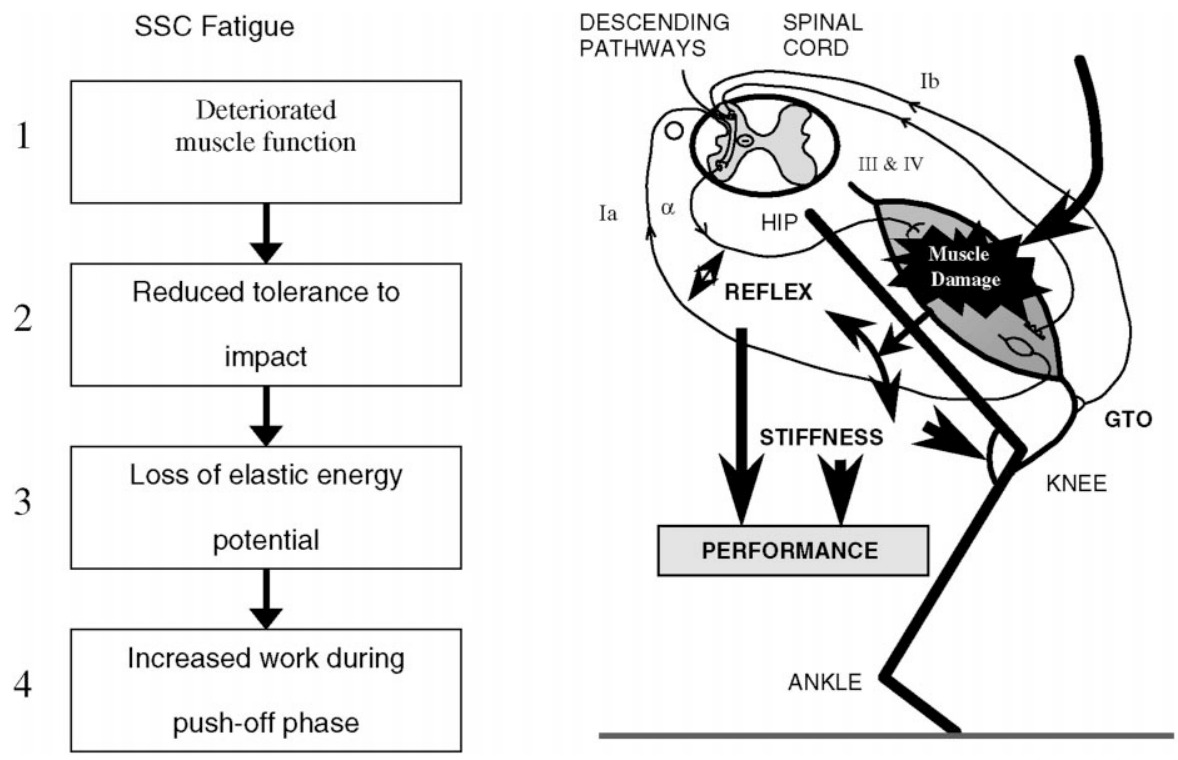

Fig. 11. Proposed coupling between SSC exercise induced muscle damage and performance reduction. Muscle damage changes stiffness regulation through changes in the afferent inputs from the muscle spindle, Golgi tendon organ and group III and IV afferent nerve endings. The events occur in the following order: 1. Due to the muscle damage the stretch-reflex sensitivity decreases; 2. Muscle (and joint) stiffness regulation becomes disturbed (reduced); 3. Efficiency of SSC function (performance) decreases. The proposed mechanism may be even more apparent in the triceps surae muscle as compared to the quadriceps group of the figure (based on Horita et al., 1999). 
or indirectly influenced by exhaustive SSC fatigue (Avela et al., 1999b). In more practical terms the sequence of events during the SSC fatiguing exercise would occur in the following order:

During exhaustive SSC exercise intrafusal fibers may themselves "fatigue" in the same manner as extrafusal fibers. However, changes recently observed in the viscous and elastic properties of the triceps surae muscle strongly suggests that the mechanical ability to stretch the muscle spindle is modified as well (Avela et al., 1999a). The potential role of deteriorated structural proteins, such as titin and desmin, in the process of muscle damage (McBride et al., 1998) and SSC fatigue has been suggested (Horita et al., 1999; Avela et al., 1999b). Projects are currently in progress to investigate this concept and preliminary results suggest that the structural integrity of titin may be compromised as the result of an exhaustive SSC exercise bout (McBride et al., work in progress). If titin break down occurs in intrafusal fibers as a result of SSC exercise performed until exhaustion it could have a direct mechanical consequence in reducing Ia afferent responsiveness.In addition, this mechanism may operate independently of the presynaptic inhibition induced by activation of the III and IV afferent nerve endings.

\section{References}

Arndt, A.N., Komi, P.V., Brüggemann, G.-P., Lukkariniemi, J., 1998. Individual muscle contributions to the in vivo achilles tendon force. Clinical Biomechanics 13, 532-541.

Avela, J., Komi, P.V., 1998a. Interaction between muscle stiffness and stretch reflex sensitivity after long-term stretch-shortening cycle (SSC) exercise. Muscle and Nerve 21, 1224-1227.

Avela, J., Komi, P.V., 1998b. Reduced stretch reflex sensitivity and muscle stiffness after long-lasting stretch-shortening cycle (SSC) exercise. European Journal of Applied Physiology 78, 403-410.

Avela, J., Kyröläinen, H., Komi, P.V. 1999a. Altered reflex sensitivity due to repeated and prolonged passive muscle stretching. Journal of Applied Physiology 86 (4), 1283-1291.

Avela, J., Kyröläinen, H., Komi, P.V., Rama, D., 1999b. Reduced reflex sensitivity persists several days after long-lasting stretch-shortening cycle (SSC) exercise. Journal of Applied Physiology, 86 (4), 1292-1300.

Belli, A., Bosco, C., 1992. Influence of stretch-shortening cycle on mechanical behaviour of triceps surae during hopping. Acta Physiologica Scandinavica 144, 401-408.

Cavagna, G.A., Dusman, B., Margaria, R., 1968. Positive work done by a previously stretched muscle. Journal of Applied Physiology 24, 21-32.

Cavagna, G.A., Saibene, F.P., Margaria, R., 1965. Effect of negative work on the amount of positive work performed by an isolated muscle. Journal of Applied Physiology 20, 157-158.

Clarkson, P.M., Nosaka, K., Braun, B., 1992. Muscle function after exercise-induced muscle damage and rapid adaptation. Medicine and Science in Sports and Exercise 24, 512-520.

Edman, K.A.P., 1980. The role of non-uniform sarcomere behaviour during relaxation of striated muscle. European Heart Journal 1 (suppl. A), 49-57.
Faulkner, J.A., Brooks, S.V., Opiteck, J.A., 1993. Injury to skeletal muscle fibers during contractions: conditions of occurence and prevention. Physio Therapy 73 (12), 911-921.

Finni, T., Komi, P.V., Lepola, V., 1998. In vivo muscle dynamics during jumping. Third Annual Congress of the European College of Sport Science. Manchester, United Kingdom, 15.-18.7.1998.

Flitney, F.W., Hirst, D.G., 1978. Cross-bridge detachment and sarcomere "give" during stretch of active frog's muscle. Journal of Physiology 276, 449-465.

Ford, L.E., Huxley, A.F., Simmons, R.M., 1978. Tension responses to sudden length change in stimulated frog muscle fibres near slack length. Journal of Physiology 269, 441-515.

Frigo, C., Pedotti, A., 1978. Determination of muscle length during locomotion. In: Asmussen, E., Jorgensen, K. (Eds.), Biomechanics VI-A. University Park Press, Baltimore, pp. 355-360.

Fukashiro, S., Komi, P.V., 1987. Joint moment and mechanical power flow of the lower limb during vertical jump. International Journal of Sports Medicine 8, 15-21.

Fukashiro, S., Komi, P.V., Järvinen, M., Miyashita, M., 1993. Comparison between the directly measured Achilles tendon force and the tendon force calculated from the ankle joint moment during vertical jumps. Clinical Biomechanics 8, 25-30.

Fukashiro, S., Komi, P.V., Järvinen, M., Miyashita, M., 1995. In vivo achilles tendon loading during jumping in humans. European Journal of Applied Physiology 71, 453-458.

Gollhofer, A., Komi, P.V., Fujitsuka, N., Miyashita, M., 1987. Fatigue during stretch-shortening cycle exercises. II Changes in neuromuscular activation patterns of human skeletal muscle. International Journal of Sports Medicine 8, 38-41.

Gregor, R.J., Komi, P.V., Browing, R.C., Järvinen, M., 1991. A comparison of the triceps surae and residual muscle moments at the ankle during cycling. Journal of Biomechanics 24, 287-297.

Gregor, R.J., Roy, R.R., Whiting, W.C., Lovely, R.G., Hodgson, J.A., Edgerton, V.R., 1988. Mechanical output of the cat soleus during treadmill locomotion in vivo vs. in situ characteristics. Journal of Biomechanics 21 (9), 721-732.

Grieve, D.W., Pheasant, S., Cavanagh, P.R., 1978. Predictions of gastrocnemius length from knee and ankle join posture. In: Asmussen, E., Jorgensen, K. (Eds.), Biomechanics VI-A. University Park Press, Baltimore, pp. 405-412.

Griffiths, R.I., 1991. Shortening of muscle fibres during stretch of the active cat medial gastrocnemius muscle: the role of tendon compliance. Journal of Physiology 436, 219-236.

Hill, A.V., 1938. The heat and shortening of the dynamic constant of muscle. Proceedings of the Royal Society London, B 126, 136-195.

Hoff, A.L., Geelen, B.A., van den Berg, J., 1983. Calf muscle moment, work and efficiency in level walkin: role of series elasticity. Jourmal of Biomechanics 16, 523-537.

Hoffer, J.A., Andreassen, S., 1981. Regulation of soleus muscle stiffness in premammillary cats. Intrinsic and reflex components. Journal of Neurophysiology 45, 267-285.

Horita, T., Komi, P.V., Nicol, C., Kyröläinen, H., 1996. Stretch-shortening cycle fatigue: interactions among joint stiffness, reflex, and muscle mechanical performance in the drop jump. European Journal of Applied Physiology 73, 393-403.

Horita, T., Komi, P.V., Nicol, C., Kyröläinen, H., 1999. Effect of exhausting stretch-shortening cycle exercise on the time course of mechanical behaviour in the drop jump: possible role of muscle damage. Eur J Appl Physiol 79, 160-167.

Huijing, P.A., 1992. Elastic potential of muscle. In: Komi, P.V. (Ed.), Strength and Power in Sport. Blackwell Scientific Pulications, Oxford, pp. 151-168.

Huxley, A.F., Simmons, R.M., 1971. Proposed mechanism of force generation in striated muscle. Nature 233, 533-538.

Van Ingen-Schenau, G.J., Bobbert, M.F., de Haan, A., 1997. Does elastic energy enhance work and efficiency in the stretch-shortening cycle? Journal of Applied Biomechanics 13, 386-415. 
Kaneko, M., Komi, P.V., Aura, O., 1984. Mechanical efficience of concentric and eccentric exercise performed with medium to fast contraction rates. Scandinavian Journal of Sport Science 6, $15-20$.

Komi, P.V., 1973. Measurement of the force-velocity relationship in human muscle under concentric and eccentric contraction. In: Jokl, E. (Ed.), Medicine and Sport, Biomechanics III, Vol. 8. Karger, Basel, pp. 224-229.

Komi, P.V., 1983. Elastic potentiation of muscles and its influence on sport performance. In: Baumann, W. (Ed.), Biomechanik und sportliche Leistung. Verlag Karl Hofmann, Schorndorf, pp. 59-70.

Komi, P.V., 1984. Physiological and biomechanical correlates of muscle function: effects of muscle structure and stretch-shortening cycle on force and speed. Exercise and Sports Sciences Reviews/ACSM 12, 81-121.

Komi, P.V., 1990. Relevance of in vivo force measurements to human biomechanics. Journal of Biomechanics 23 (suppl. 1), 23-34.

Komi, P.V., 1992. Stretch-shortening cycle. In: Komi, P.V. (Ed.), Strength and Power in Sport. Blackwell Scientific Publ, Oxford, pp. 169-179.

Komi, P.V., Belli, A., Huttunen, V., Bonnefoy, R., Geyssant, A., Lacour, J.R., 1996. Optic fibre as a transducer of a tendomuscluar forces. European Journal of Applied Physiology 72, 278-280.

Komi, P.V., Belli, A., Huttunen, V., Partio, E., 1995. Optic fiber as a transducer for direct in-vivo measurements of human tendomuscular forces. In: Häkkinen, K., Keskinen, K.L., Komi, P.V., Mero, A. (Eds.), Proceedings of XVth ISB, Jyväskylä, Finland. pp 494-495.

Komi, P.V., Gollhofer, A., 1997. Stretch reflex can have an important role in force enhancement during SSC-exercise. Journal of Applied Biomechanics 13, 451-460.

Komi, P.V., Hyvärinen, T., Gollhofer, A., Mero, A., 1986. Man-shoe-surface interaction: special problems during marathon running. Acta Universitatis Oul. A 179, 69-72.

Komi, P.V., Nicol, C., 2000. Stretch-shortening cycle fatigue. In: McIntosh, B., Nigg, B., (Eds.), Biomechanics and Biology of Movement. Human Kinetics Publ, Champaign, IL, in print.

Komi, P.V., Salonen, M., Järvinen, M., Kokko, O., 1987. In vivo registration of Achilles tendon forces in man. I. Methodological development. International Journal of Sports Medicine 8, 3-8.

Kyröläinen, H., Avela, J., Komi, P.V., 1989. Regulation of muscle and stiffness during long jump take-off. In: Gregor, R.J., Zernicke, R.F., Whiting, W.C. (Eds.), Biomechanics XII. UCLA, Los Angeles, USA, pp. 364-365.
Lee, R.G., Tatton, W.G., 1982. Long latency reflexes to imposed displacements of the human wrist. Dependence on duration of movement. Experiments in Brain Research 45, 207-216.

Luhtanen, P., Komi, P.V., 1978. Segmental contribution to forces in vertical jump. European Journal of Applied Physiology 38, 181-188.

McBride, J.M., Triplett-McBride, T., Davie, A., Abernethy, P.J., Newton, R.U., 1998. Differental expression of titin isoforms in various athletic populations. International Conference of Weightlifting and Strength Training, Lahti, Finland, November 1998.

Mero, A., Komi, P.V., 1985. Effects of supramaximal velocity on biomechanical varibles in sprinting. International Journal of Sport Biomechanics 1 (3), 240-252.

Nicol, C., Komi, P.V., 1998. Significance of passively induced stretch reflexes on achilles tendon force enhancement. Muscle and Nerve 21, 1546-1548.

Nicol, C., Komi, P.V., Avela, J., 1994. Reduced reflex sensitivity after exhaustive stretch-shortening cycle exercise (abstract). Symposium on the Neural and Neuromuscular Aspects of Muscle Fatigue, Miami, November 1994

Nicol, C., Komi, P.V., Horita, T., Kyröläinen, H., Takala, T.E.S., 1996. Reduced stretch-reflex sensitivity after exhausting stretch-shortening cycle (SSC) exercise. European Journal of Applied Physiology 72, 401-409.

Nicol, C., Komi, P.V., Marconnet, P., 1991a. Fatigue effects of marathon running on neuromuscular performance I: changes in muscle force and stiffness characteristics. Scandinavian Journal of Medicine and Science in Sports 1, 10-17.

Nicol, C., Komi, P.V., Marconnet, P., 1991b. Fatigue effects of marathon running on neuromuscular performance II: changes in force, integrated electromyographic activity and endurance capacity. Scandinavian Journal of Medicine and Science in Sports 1, 18-24.

Norman, R.W., Komi, P.V., 1979. Electromechanical delay in skeletal muscle under normal movement conditions. Acta Physiologica Scandinavica 106, 241-248.

Rack, P.M.H., Westbury, D.R., 1974. The short range stiffness of active mammalian muscle and its effect on mechanical properties. Journal of Physiology 240, 331-350.

Roberts, T.J., Marsch, R.L., Weyand, P.G., Taylor, C.R., 1997. Muscular force in running turkeys: the economy of minimizing work. Science 275, 1113-1115.

Wilkie, D.R., 1950. The relation between force and velocity in human muscle. Journal of Physiology 110, 249.

Voigt, M., Dyhre-Poulsen, P., Simonsen, E.B., 1998. Stretch-reflex control during human hopping. Acta Physiologica Scandinavica 163 (2), 181-194. 